\title{
Mathematics of Planet Earth
}

\section{Hans G. Kaper and Christiane Rousseau}

The international year MPE2013 highlighted the role of the mathematical sciences in the study of Planet Earth. Since then, Mathematics of Planet Earth (MPE) has developed into a new area of research in our discipline. MPE aims to gain, through the mathematical sciences, a better understanding of the interdependence of nature, science, and society. MPE activities focus on the state of the planet, its life-supporting capability, and the impact of human activities on the environment. MPE is more than a mathematical activity: MPE is a multidisciplinary activity par excellence for mathematical scientists. It is also an opportunity to revisit our ideas about education and training at all levels.

Following a brief summary of the origin and main themes of MPE, we present three case studies: a modeling activity to explain sea-level rise due to glacial melting, a study in progress to conceptualize the notion of resilience, and an ongoing effort to explore the effects of a changing climate on food security.

\section{The Origin of MPE}

The idea of MPE was conceived in 2009, when the North American mathematical research institutes were exploring options for long-term collaborations. Plans were made to organize a yearlong program of scientific activities, including workshops, conferences, and educational and public outreach activities, to be launched in 2013. An introductory year was held in 2010-11 called Climate Change and Sustainability.

Hans G. Kaper is a SIAM Fellow affiliated with Georgetown University. His email address is hans. kaper@georgetown.edu.

Christiane Rousseau is an AMS Fellow and a retired professor of mathematics at Université de Montréal. Her email address is rousseac@dms . umontrea1 .ca.

For permission to reprint this article, please contact: reprint-permission aams.org.

DOI: https://dx.doi.org/10.1090/noti 1977
In the United States, the planning effort was coordinated by the American Institute of Mathematics (AIM). The program went international at the International Congress of Mathematicians in 2010 (ICM 2010) and received the patronage of UNESCO in 2012. It became known as "MPE2013." 1

After ICM 2010, word spread quickly, and the MPE logo (see Figure 1) could be seen around the world.

Many organizations joined the effort; eventually, MPE2013 involved fifteen long-term programs at mathematical research institutes, sixty workshops, dozens of special sessions at society meetings, two daily blogs (one in English, one in French), several public lecture series, summer and winter schools for graduate students, research experiences for undergraduates, open-source curriculum materials for all ages and grades, ${ }^{2}$ and a juried international competition to produce an Open Source Exhibition. The exhibition is hosted by IMAGINARY ${ }^{3}$ and has been shown at many venues around the world. Anthologies of the French and English blog posts were published in [1] and [2],

respectively.

\section{MPE after 2013}

At the end of 2013, MPE2013 morphed into MPE, which has been the brand name since then. The idea of integrating the mathematical sciences-including mathematics, statistics, operations research, and computational and data science-into the study of our planet, linking nature, science, and society, is now firmly embedded in the fabric of research and education.

\footnotetext{
$1 \longdiv { \text { dimacs.rutgers.edu/archive/mpe_2013 } }$

${ }^{2}$ Some of these materials still exist at www.mathofplanetearth.org /curriculum-material.

3 https://imaginary.org
} 
The DIMACS Center at Rutgers University, with support from the National Science Foundation, organized a series of 23 workshops over a period of five years (MPE2013+), some of which were in a cluster called "Education for the Planet Earth of Tomorrow" and all of which included sessions on educational issues. ${ }^{4}$ The Society for Industrial and Applied Mathematics (SIAM) created a SIAM Activity Group on Mathematics of Planet Earth (SIAG/MPE). Its biennial meetings offer a forum for ongoing research and education projects in MPE. ${ }^{5}$ The SIAG/MPE Prize, which recognizes either significant scientific work in topic areas that are relevant to MPE or sustained or seminal contributions to the scientific agenda of the SIAG/MPE, will be awarded for the first time at MPE20. The Engineering and Physical Sciences Research Council (EPSRC) in the UK funded a Center for Doctoral Training at Imperial College London and the University of Reading (MPE-CDT), with a new degree program in MPE. ${ }^{6}$ In the Netherlands, NWO (counterpart of the United States NSF) supports a research program in MPE as part of its Nonlinear Dynamics of Natural Systems cluster NDNS+. ${ }^{7}$ The Mathematics and Climate Research Network (MCRN) continues its research and training programs in the United States. ${ }^{8}$ Many groups and individual researchers around the world pursue research in informal settings on topics related to MPE.

\section{The Success of MPE2013}

Why was MPE2013 successful, and why did it spread like wildfire around the world? Certainly the idea was timely. Humanity faces major global challenges: adapting to a changing climate, protecting the environment, maintaining biodiversity, feeding a rapidly increasing population, and encouraging sustainable development while ensuring equity for all are just a few among many. The younger generation, our students, see the urgency of these challenges, and they are pushing for action. MPE offers them an entry point to get involved.

MPE2013 demonstrated that MPE is a great way to show the relevance of the mathematical sciences. It stimulated interest in our discipline among scientists, in schools, and among the general public.

The model of MPE2013 was new. MPE2013 was a grassroots organization with clearly defined goals: on the one hand, to open up new areas of research in the mathematical sciences, to attract a new generation of scientists through education and training, and to stimulate a culture of mul-

\footnotetext{
4 dimacs.rutgers.edu/programs/sf/mpe2013-plus

https://www.siam.org/membership/Activity-Groups/detail /mathematics-of-planet-earth

mpecdt.org

7 https://www.nwo.n1/en/research-and-resu1ts/programmes /mathematics+clusters

8 https://mcrn.hubzero.org
}

tidisciplinary collaborations; on the other hand, to inform the public, the media, and the educational establishments of the essential role that the mathematical sciences play in addressing planetary challenges. MPE2013 had no budget; it functioned on a global scale with some 140 partners worldwide (research institutes, scientific societies, universities, and foundations) who committed to organize international, national, and local activities with local funding.

Surely, a distinctive feature of MPE2013 was the extraordinary and unprecedented level of global collaboration. It illustrated brilliantly that by joining forces we can achieve great things and hope for a significant impact.

\section{The Themes of MPE}

MPE activities are commonly grouped under four themes, considering Planet Earth as a physical system, as a system supporting life, as a system organized by humans, and as a system at risk. The boundaries are not sharp but provide an organizing principle for the many topics that fall under the MPE umbrella.

Planet Earth as a physical system encompasses topics like Earth's climate system, weather and climate, natural resources, and sustainability. Planet Earth as a system supporting life includes the carbon cycle, population dynamics, the spread of infectious diseases, mathematical ecology, biodiversity, and evolution. Human activities are central to Planet Earth as a system organized by humans, covering topics from land use to urban infrastructure, energy, water, transportation, ecosystem services, food security, and socio-economics. Lastly, a planet at risk includes statistics of extreme events, attribution of such events to climate change, issues of vulnerability and resilience, and risks associated with invasive species and natural disasters.

When nature and humans interact, a complex system results. Instead of natural laws and fundamental equations, we are dealing with collective behavior, feedback mechanisms, information flows, and human judgment calls. Much of the research is data-driven and motivated by decision-makers' concerns. This is a new world for the mathematical sciences. Integrating these disparate elements into the language of mathematics is a major challenge for MPE.

\section{What Makes MPE Interesting?}

As a multidisciplinary activity, MPE reaches far beyond the boundaries of the mathematical sciences. It provides real opportunities to address some of the challenges facing our planet.

MPE covers a broad range of topics. Table 1 shows the titles of the chapters of a forthcoming book on MPE [3]; the list is representative but certainly not exhaustive. Topics such as population dynamics and the spread of infectious diseases are well established in the mathematical research community, but the simple models of old no longer match 
the current trend toward data-driven modeling using artificial intelligence and machine-learning [3].

\section{Mathematics of the Not-So-Solid Solid Earth}

We use models to forecast the future. One way to test a model is to use it to reconstruct the past, for which we have data. The data show that sea levels have fluctuated significantly in the past. An explanation comes from the alternation of periods of glaciation and deglaciation. In a period of deglaciation, glaciers melt and water that is stored in them is added to that of the oceans. But data suggest that things are not so simple. In Amsterdam, the sea level increased nearly $200 \mathrm{~mm}$ in the period from 1700 to 1925 , while in Stockholm it decreased almost $1000 \mathrm{~mm}$. Why? The chapter shows how we need to include more and more ingredients into a model to explain the phenomenon.

The first ingredient is the definition of sea level. We are used to thinking of sea level as altitude zero and altitude as the distance of a point to the center of the earth. But the earth is not a sphere, only a flattened geoid. Geodesists define altitude zero through a mathematical concept, namely, as a level surface of the gravitational field. At the time of the last glaciation, there was a very thick mass of ice over the polar regions, much closer to Stockholm than to Amsterdam. This ice mass exerted a gravitational attraction on the seawater; as a result, the level was higher in Stockholm than in Amsterdam. This gravitational pull disappeared with

\section{Chapter titles from [3].}

1. Modeling the Dynamics of Glacial Cycles

2. Mathematics of the Not-So-Solid Solid Earth

3. Mathematical Challenges in Measuring Variability Patterns for Precipitation Analysis

4. Mathematics of Malaria and Climate Change

5. A Risk-Structured Mathematical Model of Buruli Ulcer Disease in Ghana

6. Data-Informed Modeling in the Health Sciences

7. Multistability in Ecosystems: Concerns and Opportunities for Ecosystem Function in Variable Environments

8. Measurement of Biodiversity: Richness and Evenness

9. The Mathematics of Extinction Across Scales: From Population to the Biosphere

10. Modeling Food Systems

11. Dynamic Optimization, Natural Capital, and Ecosystem Services

12. Qualitative Models for Infrastructure Restoration after Extreme Events: Network Optimization Meets Scheduling

13. The Internet of Things and Machine Learning Solutions for Urban Infrastructure Management the melting of the glaciers, thus leading to the decrease in Stockholm in parallel with a global increase of the quantity of ocean water. But there is more to the phenomenon. We are used to thinking of the earth as a solid, but the chapter invites us to model it as a fluid (a very viscous fluid, of course) through the Navier-Stokes equations! In fact, again, this model is still too basic. Earth is viscoelastic. The weight of thick glaciers deforms the surface of the earth, and there is an elastic bounce when the weight disappears with the melting of the glacier.

Each ingredient comes from a different area of mathematics. As is typical with MPE problems, there are many ingredients at play. The challenge is to understand which ones are the most relevant and to integrate them into a complex model. We invite you to read this chapter for more details.

\section{Resilience in the Digital Age}

Multidisciplinary collaborations need time to mature. There are language barriers and cultural differences, and progress can be slow. This is a challenge for MPE.

An exciting opportunity presented itself in 2017, when one of us (CR) met Amy Luers at the General Assembly of the International Council of Science (ICSU) in Taipei. Luers is the executive director of Future Earth and former assistant director for climate resilience and information at the White House Office of Science and Technology Policy (OSTP).

Future Earth is an international nonprofit organization with the mission to accelerate transformations to global sustainability through research and innovation. ${ }^{9}$ Luers, who has a PhD in environmental science, was interested in starting an interdisciplinary research network on Resilience in the Digital Age. The notion of resilience is intuitively clear: the ability of a system to recover from a disturbance. However, the concept needs to be made operational to be of practical value. The project seemed a good match and raised immediate enthusiasm in the MPE community. The international network of Future Earth brings expertise in economics and ecology to the collaboration; on the MPE side, we offer an international network of mathematical scientists with many years of experience in mathematical and statistical modeling and data science. The collaboration brings MPE one step closer to decision-makers and strengthens our efforts to focus on societally relevant problems.

Together with Future Earth, DIMACS, and the Centre de Recherches Mathématiques (CRM), the MPE network has initiated an international collaboration to address fundamental questions, including (i) how to define resilience mathematically, (ii) how to measure resilience, and (iii) how to operationalize resilience for complex systems. In the initial phase, we are considering three particular sys- 
tems: namely, ecological systems, urban systems, and food systems. The goal is to come to a common understanding, develop a methodology for assessing resiliency, and, if possible, design a set of common standards.

\section{Food Systems and Food Security}

Food security is being recognized as a major issue in the not-too-distant future. The global population is expected to increase from close to seven billion people today to approximately ten billion people by 2050; an increasing fraction of the population will be living in urban environments, far from food-producing areas; the natural resources are finite; a changing climate affects where food can be grown; invasive species and diseases may increase the risk of crop failure. Can we use mathematical models and their predictive capabilities to explore options and assess future courses of action?

Food security prevails when all members of a community, at all times, have access to enough food for an active and healthy life; a food system is the means to achieve food security. A food system must be sustainable, equitable, and resilient to external disturbances.

A food system can be local, regional, or global and cover one or more food groups. Basically, it is a supply chain operating under biophysical, environmental, and socio-economic constraints. Its agents are producers, who grow foodstuffs, raise animals, etc.; food-chain actors, who process, ship, or sell food products; and consumers, who shop for food and prepare it for consumption. Waste occurs at all levels; some of it is preventable, much of it is not, unless additional energy is spent.

Food systems have traditionally been considered from the perspective of economics. But there is much more at play than economics, and this is where MPE comes in. For example, what would a mathematical model of a fair, equitable, and resilient food distribution system look like? Moving to sustainability will require significant changes in the food systems. Can we use mathematical techniques to guide the necessary transitions? How do we balance monetary values, nutritional values, and consumer satisfaction with the need to feed the world's population in ways that are sustainable, equitable, and resilient? Recurring periods of drought increase the likelihood of desertification-the transition from arable land to desert. What are the warning signs? Can we use ideas from the theory of dynamical systems to gain insight into critical points, where the system can experience drastic changes; or tipping points, where it can experience irreversible changes; or hysteresis, where it can experience reversible changes but the reversal requires significant resources? More generally, a warming climate and changing precipitation patterns will affect agricultural production. Can we express the connection through conceptual models and explore future scenarios?

\section{How to Get Involved in MPE}

As scientists and informed citizens, we value evidence-based decision-making. We know that human activities and global change threaten our environment, if not in the immediate future, then certainly in the coming decades. We have a responsibility to get involved and apply the particular skills of our discipline to address the challenges facing our planet.

Fortunately, we are also in a position to choose how we wish to get involved. We may decide to pursue research questions and train the next generation of researchers, we may use MPE examples to enrich our teaching or as projects for students, we may get involved in informing the public and students about the role that the mathematical sciences can play in addressing some of the challenges facing our planet. No matter your choice, there is work to be done. We urge you to get involved!

\section{References}

[1] Andler M, Bel L, Benzoni S, Goudon T, Imbert C, Rousseau A. Brèves de Maths: Mathématiques de la planète Terre, Nouveau Monde éditions, 2014.

[2] Kaper HG, Rousseau C. Mathematics of Planet Earth: Mathematicians Reflect on How to Discover, Organize, and Protect Our Planet, SIAM, Philadelphia, PA, 2015.

[3] Kaper HG, Roberts FS (eds.). Mathematics of Planet Earth: Protecting Our Planet, Learning from the Past, Safe-guarding the Future, Springer Verlag, 2019, to appear.

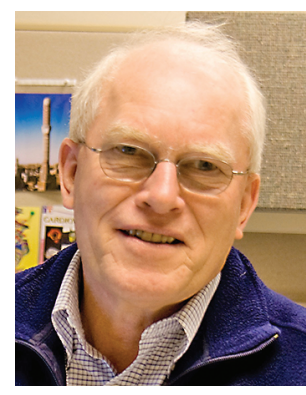

Hans G. Kaper

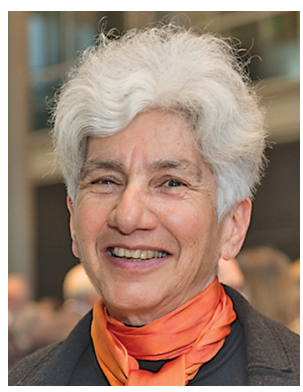

Christiane Rousseau

\section{Credits}

Photo of Hans G. Kaper is courtesy of Hans G. Kaper.

Photo of Christiane Rousseau is courtesy of Amélie Philibert, University of Montréal. 